\title{
Cytokines, chemokines and soluble adhesion molecules in aqueous humor of children with uveitis
}

\author{
Karen M. Sijssens ${ }^{\text {a,*}}$, Ger T. Rijkers ${ }^{b}$, Aniki Rothova ${ }^{\text {a }}$, Jan S. Stilma ${ }^{\text {a }}$, Peter \\ A.W.J.F. Schellekens ${ }^{a}$, Joke H. de Boer ${ }^{a}$ \\ ${ }^{\text {a }}$ F.C. Donders Institute of Ophthalmology, University Medical Center Utrecht, E.03.136, Heidelberglaan 100, 3584 CX Utrecht, The Netherlands \\ ${ }^{\mathrm{b}}$ Department of Pediatric Immunology, Wilhelmina Children's Hospital, University Medical Center Utrecht and Department of Medical \\ Microbiology and Immunology, St. Antonius Hospital, Nieuwegein, The Netherlands
}

Received 19 January 2007; accepted in revised form 10 June 2007

Available online 22 June 2007

\begin{abstract}
Uveitis in childhood is a visual threatening disease with a complication rate of more than $75 \%$. Despite extensive research, the etiology of uveitis is still unclear although the general opinion is now that uveitis is a T-cell mediated disease. The purpose of this study was to investigate the profile of cytokines, chemotactic cytokines (chemokines) and soluble adhesion molecules in the aqueous humor (AqH) of children with uveitis in order to identify the factors that control the immune response in the eye. In this clinical laboratory investigation we analyzed, with a multiplex immunoassay, 16 immune mediators in the $\mathrm{AqH}$ of 25 children with uveitis and 6 children without uveitis. Increased levels of interleukin-2 (IL-2), IL-6, IL-10, IL-13, IL-18, interferon- $\gamma$, tumor necrosis factor- $\alpha$, soluble intercellular adhesion molecule-1, RANTES, IL- 8 and interferon-inducible 10-kDa protein were found in the $\mathrm{AqH}$ of children with uveitis compared with controls. No significant differences were found for IL-1 $\beta$, IL-4, IL-12 p-70, soluble vascular cell adhesion molecule 1 and Eotaxin. Lower levels of IL-10 and IL-8 were found in quiet stage uveitis (surgical) samples compared with active uveitis (diagnostic) samples and in samples of patients treated with methotrexate (MTX) compared with samples of patients not treated with MTX. Lower levels of IL-10 were as well found in samples taken during the first 3 months after the diagnosis of uveitis than samples taken later during the disease process. No significant differences were found between patients treated with or without topical or systemic (perioperative and long term) corticosteroids. In conclusion, in children with uveitis, multiple intraocular cytokines, chemokines and soluble adhesion molecules are increased in the AqH regardless of active or inactive inflammation. Whether the IL-8 and IL-10 levels in AqH of children with uveitis are correlated with uveitis activity, early or late phase of the course of the disease and systemic treatment with MTX needs further investigation in a bigger study population.
\end{abstract}

(C) 2007 Elsevier Ltd. All rights reserved.

Keywords: uveitis; childhood; aqueous humor; cytokines; chemokines; soluble adhesion molecules; juvenile idiopathic arthritis; methotrexate

\section{Introduction}

Uveitis in childhood is a visual threatening disease with a complication rate of more than $75 \%$ (de Boer et al., 2003). Despite extensive research, the etiology of uveitis is still unclear although the general opinion is now that uveitis is a Tcell mediated disease (Lacomba et al., 2000). Several studies

\footnotetext{
* Corresponding author. Tel.: +3130250 7880; fax: +31302505417. E-mail address: k.sijssens@umcutrecht.nl (K.M. Sijssens).
}

have shown that several cytokine levels are elevated in the aqueous humor $(\mathrm{AqH})$ of patients with uveitis (Curnow et al., 2005; Takase et al., 2006). However, these studies are based on adult study populations but the immune mediator profile in $\mathrm{AqH}$ of childhood uveitis is unknown. Uveitis in childhood differs in several aspects of that in adults. The clinical presentation, underlying cause and complications of uveitis differs between children and adults (Kanski and Shun-Shin, 1984; Rothova et al., 1996; Tugal-Tutkun et al., 1996; de Boer et al., 2003; Evereklioglu, 2005). Whether these differences reflex the cytokine profile in $\mathrm{AqH}$ is not yet clear. 
The purpose of this study was to investigate the profile of cytokines, chemotactic cytokines (chemokines) and soluble adhesion molecules in the $\mathrm{AqH}$ of children with uveitis in order to identify the factors that control the immune response in the eye of this young population.

\section{Materials and methods}

\subsection{Patients}

This research followed the tenets of the Declaration of Helsinki and was approved by our institutional review board. Written informed consent was obtained from each child and/ or parent after explanation of the nature of the study.

$\mathrm{AqH}$ samples were collected as prescribed previously, stored immediately at $-80{ }^{\circ} \mathrm{C}$ in sterile screw-cap tubes and thawed directly before analysis within 4 years of collection (van der Lelij and Rothova, 1997). This technique is to preserve the sample for analysis without degradation. AqH samples of 25 children with uveitis under the age of 16 were used. These samples were obtained between 2002 and 2005 during surgery for cataract $(n=8)$ or glaucoma $(n=8)$. In the perioperative period patients were treated with systemic corticosteroids $(1 \mathrm{mg} / \mathrm{kg})$ starting 1 day to 1 week before surgery $(n=9)$, long term systemic corticosteroids $(n=3)$ or no systemic corticosteroids $(n=4)$. The remaining 9 samples were collected for diagnostic purposes. These 9 samples were analyzed for herpes simplex virus, varicella zoster virus and Toxoplasma gondii antibodies and DNA by PCR (de Groot-Mijnes et al., 2006). The clinical data of all patients are shown in Table 1. AqH samples of 6 children under the age of 16 , who had an operation for congenital cataract $(n=3)$, glaucoma not associated with uveitis $(n=2)$ or lens subluxation due to Marfan's syndrome $(n=1)$, were collected as controls.

In 6 children with uveitis (nos. 3, 7, 8, 17, 18 and 25), more than one sample was available of the same eye. We compared in these 6 pairs of samples the levels of cytokines, chemokines and soluble adhesion molecules before and after surgery.

We recorded the following clinical data for each patient: age at diagnosis of uveitis, age at the time of sample collection, localization and course of uveitis (acute or chronic), underlying systemic disease and (systemic) treatment at the time of sample collection (Table 1).

\subsection{Immunoassay}

We analyzed the AqH samples in a multiplex immunoassay as described previously (de Jager et al., 2005). In each 50- $\mu$ l sample, we analyzed 16 mediators; interleukin- $1 \beta$ (IL-1 $\beta$ ), IL-2, IL-4, IL-6, IL-10, IL-12 p-70, IL-13, IL-18, interferon$\gamma($ IFN- $\gamma$ ), tumor necrosis factor- $\alpha$ (TNF- $\alpha$ ), soluble intercellular adhesion molecule 1 (sICAM-1; CD54), soluble vascular cell adhesion molecule 1 (sVCAM-1; CD106), RANTES (Regulated on Activation, Normal T-cell Expressed, and Secreted; CCL5), Eotaxin (CCL11), IL-8 (CXCL8) and interferon-inducible 10-kDa protein (IP-10; CXCL10). Concentrations above or below the detection limit were given as the highest or lowest detectable value. For statistical analysis concentrations below the detection limit were converted to a value of $0.5 \times$ the lowest point of the calibration curve.

\subsection{Statistical analysis}

Statistical analysis of the data was performed by using the SPSS statistical software package version 12.0.1 (SPSS Inc., Chicago, IL). The Kruskal-Wallis and the Mann-Whitney $U$-tests were used to compare the means of the (non-parametric) different groups. The Pearson Chi-square test or Fisher's exact test was used to compare possible associations between categorical variables where appropriate. We used the Wilcoxon Signed Rank test to compare paired samples. $p$ values of less than. 05 were considered to be statistically significant.

\section{Results}

Aqueous humor was collected from 25 children with uveitis associated with juvenile idiopathic arthritis (JIA; $n=11$ ), herpes virus infection $(n=1)$, Fuchs' heterochromic cyclitis $(n=1)$, HLA-B27 $(n=2)$, Devic's neuromyelitis optica (NMO; $n=1)$ or of unknown etiology $(n=9$, Table 1$)$. The child with a herpes virus infection had a keratouveitis and the diagnosis was confirmed by a positive PCR for varicellazoster virus in AqH. Both patients with HLA-B27 positive uveitis had acute alternating anterior uveitis. The mean age at sample collection was 9.6 years (range 5.2-14.8 years) for children with uveitis and 3.3 years (range $0.2-9.5$ years, $p=0.002$ ) for the control patients. The mean duration of uveitis till sampling was 2.3 years (median: 1.4 years, range: $0-$ 9 years).

Significantly higher levels of IL-2, IL-6, IL-10 IL-13, IL18 , IFN- $\gamma$, TNF- $\alpha$, sICAM-1, RANTES, IL-8 and IP-10 were found in children with uveitis than in children without uveitis (Table 2). The levels of IL-1 $\beta$, IL-4, IL-12 p-70, sVCAM-1 and Eotaxin did not significantly differ in AqH of uveitis compared with controls (Table 2).

No significant differences were found between anterior $(n=16)$ and intermediate $(n=5)$ uveitis for all the 16 mediators studied. There were only 2 children with posterior uveitis and 2 children with panuveitis therefore no comparison was made between all 4 anatomic classifications. Furthermore, between samples from chronic $(n=21)$ and acute $(n=4)$ uveitis no significant differences were found for all 16 mediators, but levels of IP-10 and IL-2 tended to be higher in chronic uveitis ( $1013 \mathrm{pg} / \mathrm{ml}$ versus $361 \mathrm{pg} / \mathrm{ml} ; p=0.068$ and $47 \mathrm{pg} / \mathrm{ml}$ versus $5 \mathrm{pg} / \mathrm{ml} ; p=0.081$ respectively).

Treatment with or without topical corticosteroids had no influence on the mediator levels in AqH of children with uveitis ( $n=20$ versus $n=5$, respectively). Both groups were equally treated with systemic corticosteroids (perioperative or long term) and with MTX ( $p=0.821$ and $p=0.341$, respectively). Cytokine levels of children treated with perioperative corticosteroids $(n=9)$ did not differ significantly from those not treated with systemic corticosteroids $(n=12)$. These two groups did not differ in treatment with topical corticosteroids 
Table 1

Clinical data of all uveitis patients

\begin{tabular}{|c|c|c|c|c|c|c|c|c|c|c|c|}
\hline No. & Gender & $\begin{array}{l}\text { Age at } \\
\text { diagnosis } \\
\text { of uveitis } \\
\text { (years) }\end{array}$ & $\begin{array}{l}\text { Age at } \\
\text { aqueous } \\
\text { humor sample } \\
\text { collection } \\
\text { (years) }\end{array}$ & $\begin{array}{l}\text { Localization } \\
\text { of uveitis }\end{array}$ & $\begin{array}{l}\text { Underlying } \\
\text { cause of } \\
\text { uveitis }\end{array}$ & $\begin{array}{l}\text { Course of } \\
\text { uveitis }\end{array}$ & $\begin{array}{l}\text { Sample } \\
\text { type }\end{array}$ & $\begin{array}{l}\text { Sample taken } \\
\text { during the first } \\
3 \text { months after the } \\
\text { diagnosis of uveitis }\end{array}$ & $\begin{array}{l}\text { Topical } \\
\text { corticosteroids }\end{array}$ & $\begin{array}{l}\text { Systemic } \\
\text { corticosteroids }\end{array}$ & $\begin{array}{l}\text { MTX } \\
\text { (mg/week) }\end{array}$ \\
\hline 1 & $\mathrm{~F}$ & 3.9 & 12.8 & Anterior & JIA & Chronic & Surgical $^{\mathrm{b}}$ & No & Yes & Perioperative & 10 \\
\hline 2 & M & 4.1 & 5.5 & Anterior & JIA & Chronic & Surgical $^{\mathrm{b}}$ & No & Yes & No & 7.5 \\
\hline \multirow[t]{2}{*}{$3^{\mathrm{a}}$} & $\mathrm{F}$ & 4.5 & 12.8 & Anterior & JIA & Chronic & Surgical $^{\mathrm{b}}$ & No & Yes & No & 10 \\
\hline & & & & & & & Surgical $^{\mathrm{c}}$ & No & No & Perioperative & 10 \\
\hline 4 & $\mathrm{~F}$ & 3.9 & 5.6 & Anterior & JIA & Chronic & Surgical $^{\mathrm{b}}$ & No & Yes & Perioperative & 0 \\
\hline 5 & $\mathrm{~F}$ & 1.9 & 5.2 & Anterior & JIA & Chronic & Surgical $^{\mathrm{b}}$ & No & Yes & Perioperative & 20 \\
\hline 6 & $\mathrm{~F}$ & 9.4 & 11.4 & Anterior & JIA & Chronic & Surgical $^{\mathrm{c}}$ & No & Yes & No & 10 \\
\hline \multirow[t]{2}{*}{$7^{\mathrm{a}}$} & M & 3.8 & 7.5 & Anterior & JIA & Chronic & Surgical $^{\mathrm{c}}$ & No & Yes & Long term & 7.5 \\
\hline & & & & & & & Surgical $^{\mathrm{b}}$ & No & Yes & Perioperative & 10 \\
\hline \multirow[t]{2}{*}{$8^{\mathrm{a}}$} & $\mathrm{F}$ & 6.4 & 10.6 & Anterior & JIA & Chronic & Surgical $^{\mathrm{c}}$ & No & Yes & Long term & 0 \\
\hline & & & & & & & Surgical $^{\mathrm{c}}$ & No & Yes & Perioperative & 0 \\
\hline 9 & $\mathrm{~F}$ & 7.0 & 12.3 & Anterior & JIA & Chronic & Surgical $^{\mathrm{c}}$ & No & Yes & Perioperative & 10 \\
\hline 10 & M & 5.9 & 9.3 & Anterior & JIA & Chronic & Surgical $^{\mathrm{c}}$ & No & Yes & Perioperative & 7.5 \\
\hline 11 & $\mathrm{~F}$ & 3.5 & 10.4 & Anterior & JIA & Chronic & Surgical $^{\mathrm{c}}$ & No & Yes & Perioperative & 7.5 \\
\hline 12 & $\mathrm{~F}$ & 14.4 & 14.4 & Anterior & HLA-B27 & Acute & Diagnostic & Yes & Yes & No & 0 \\
\hline 13 & M & 10.4 & 10.4 & Anterior & HLA-B27 & Acute & Diagnostic & Yes & Yes & No & 0 \\
\hline 14 & M & 5.9 & 6.0 & Anterior & Herpes virus ${ }^{\mathrm{d}}$ & Acute & Diagnostic & Yes & No & No & 0 \\
\hline 15 & M & 14.6 & 14.8 & Anterior & Fuchs' & Chronic & Surgical $^{\mathrm{b}}$ & Yes & Yes & Perioperative & 0 \\
\hline 16 & $\mathrm{~F}$ & 7.8 & 9.2 & Anterior & Eci & Chronic & Surgical $^{\mathrm{c}}$ & No & Yes & No & 7.5 \\
\hline \multirow[t]{2}{*}{$17^{\mathrm{a}}$} & $\mathrm{F}$ & 10.1 & 12.2 & Intermediate & Eci & Chronic & Surgical $^{\mathrm{c}}$ & No & Yes & Perioperative & 0 \\
\hline & & & & & & & Surgical $^{\mathrm{b}}$ & No & Yes & Perioperative & 12.5 \\
\hline \multirow[t]{2}{*}{$18^{\mathrm{a}}$} & M & 9.2 & 9.2 & Intermediate & Eci & Chronic & Diagnostic & Yes & Yes & No & 0 \\
\hline & & & & & & & Surgical $^{\mathrm{b}}$ & No & Yes & Perioperative & 0 \\
\hline 19 & $\mathrm{~F}$ & 10.5 & 10.7 & Intermediate & Eci & Chronic & Diagnostic & Yes & Yes & No & 0 \\
\hline 20 & M & 6.1 & 6.1 & Intermediate & Eci & Chronic & Diagnostic & Yes & No & No & 0 \\
\hline 21 & M & 5.3 & 5.4 & Intermediate & Eci & Chronic & Diagnostic & Yes & Yes & No & 0 \\
\hline 22 & M & 5.4 & 5.4 & Posterior & NMO & Acute & Diagnostic & Yes & No & No & 0 \\
\hline 23 & M & 6.7 & 6.8 & Posterior & Eci & Chronic & Surgical $^{\mathrm{b}}$ & Yes & No & Perioperative & 0 \\
\hline 24 & $\mathrm{~F}$ & 11.2 & 14.0 & Panuveitis & Eci & Chronic & Surgical $^{\mathrm{b}}$ & No & No & Long term & 20 \\
\hline \multirow[t]{2}{*}{$25^{\mathrm{a}}$} & $\mathrm{F}$ & 12.7 & 13.1 & Panuveitis & Eci & Chronic & Diagnostic & No & Yes & Long term & 0 \\
\hline & & & & & & & Surgical $^{\mathrm{c}}$ & No & Yes & Long term & 0 \\
\hline
\end{tabular}

MTX, methotrexate; JIA, juvenile idiopathic arthritis; NMO, neuromyelitis optica/Devic's syndrome.

${ }^{\text {a }}$ For these patients 2 successive samples were available.

${ }^{b}$ Cataract surgery.

c Glaucoma surgery.

d Varicella-zoster virus.

and MTX ( $p=0.603$ and $p=0.396$, respectively). Treatment with long-term systemic corticosteroids $(n=4)$ or no systemic corticosteroids $(n=12)$ at time of sample collection had no influence on the mediator levels in the $\mathrm{AqH}$ of children with uveitis. Both groups were equally treated with topical corticosteroids and with MTX ( $p=1.000$ and $p=0.604$, respectively). Children treated with MTX $(n=11)$ at time of sample collection showed significantly lower levels of IL-8 (24 pg/ml versus $102 \mathrm{pg} / \mathrm{ml}$, respectively, $p=0.021$ ) and IL$10(3 \mathrm{pg} / \mathrm{ml}$ versus $11 \mathrm{pg} / \mathrm{ml}, p=0.008)$ than children not treated with MTX ( $n=14$, Table 3$)$. Both groups did not differ significantly in systemic (perioperative or long term) and topical treatment with corticosteroids $(p=0.567$ and $p=0.341$, respectively). Of all children treated with MTX, $9(9 / 11,82 \%)$ had JIA-associated uveitis and $2(2 / 11,18 \%)$ had chronic uveitis of unknown etiology; all samples were collected during surgery. The mean duration of MTX treatment before sample collection was 2.2 years (median: 1.6 years, range: $0.2-8.4$ years) with a mean dose of $10 \mathrm{mg} /$ week (median: $10.6 \mathrm{mg} /$ week, range: $7.5-20 \mathrm{mg} /$ week).

When comparing samples from children before any kind of systemic treatment (corticosteroids and/or MTX) was started $(n=8)$ with samples from children who were already systemically treated $(n=17)$, significantly higher levels of IL-8 were found in the first group $(129 \mathrm{pg} / \mathrm{ml}$ versus $36 \mathrm{pg} / \mathrm{ml}$, $p=0.019$ ) and IL-10 tended to be higher in that group (13 $\mathrm{pg} / \mathrm{ml}$ versus $4 \mathrm{pg} / \mathrm{ml}, p=0.097)$.

Significantly lower levels of IL-10 were observed in patients with quiet stage uveitis samples (collected during surgery, $n=16$ ) compared with active uveitis samples (collected for diagnostic purposes, $n=9 ; 3 \mathrm{pg} / \mathrm{ml}$ versus $15 \mathrm{pg} / \mathrm{ml} ; p=0.027)$. Also, IL-8 was found to be lower in quiet uveitis samples $(31 \mathrm{pg} / \mathrm{ml}$ versus $144 \mathrm{pg} / \mathrm{ml}$; $p=0.004)$. Significantly more children had JIA-associated uveitis in the quiet uveitis group (11/16) than children in the active phase of the disease $(0 / 9, p=0.001)$. Children with 
Table 2

Cytokine, chemokine and adhesion molecule levels in aqueous humor of children with uveitis and in children with juvenile idiopathic arthritis associated uveitis compared with controls

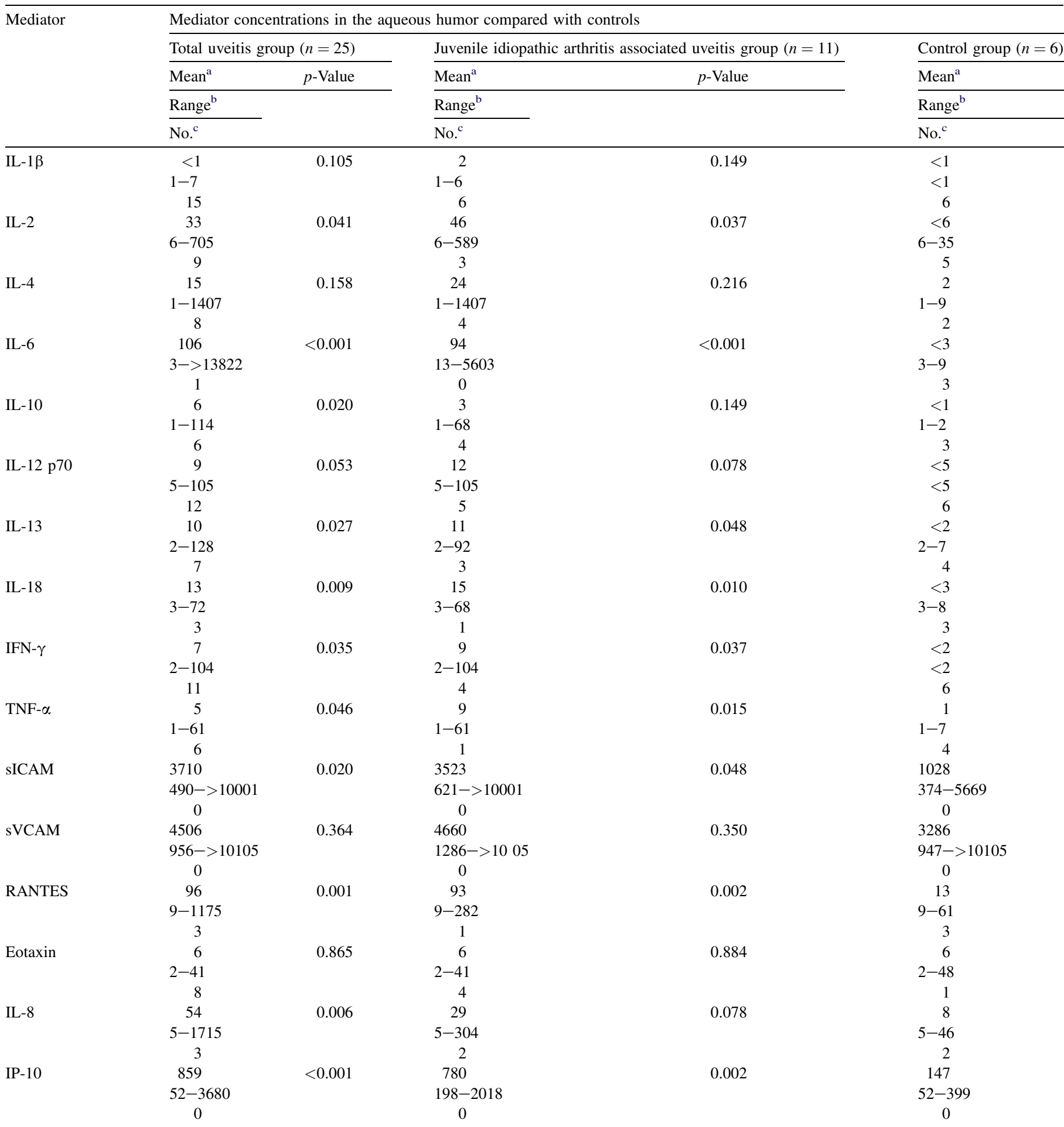

IL, interleukin; IFN, interferon; TNF, tumor necrosis factor; sICAM, soluble intercellular adhesion molecule; sVCAM, soluble vascular cell adhesion molecule; RANTES, regulated on activation normal T-cell expressed and secreted; IP, interferon-inducible protein.

Sensitivity of the assay is $1.4 \mathrm{pg} / \mathrm{ml}$ for IL-1 $\beta, 1.8$ for IL-2, 1.2 for IL-4, 2.4 for IL-6, 2.3 for IL-10, 4.3 for IL-12 p70, 1.0 for IL-13, 1.2 for IL-18, 9.1 for IFN- $\gamma$, and 1.2 for TNF- $\alpha$. Sensitivity for sICAM (CD54) is $26.4 \mathrm{pg} / \mathrm{ml}$, for sVCAM (CD106) 22.3, for RANTES (CCL5) 1.5, for Eotaxin (CCL11) 1.3, for IL-8 (CXCL8) 5.3, and for IP-10 (CXCL10) 1.0 (sensitivity data from de Jager et al., 2005).

a Geometric mean concentration $(\mathrm{pg} / \mathrm{ml})$.

b Range of detectable measured samples (pg/ml).

c Number of samples in the undetectable range. 
Table 3

Cytokine, chemokine and adhesion molecule levels in aqueous humor of children with uveitis treated with methotrexate compared with children with uveitis not treated with methotrexate

\begin{tabular}{|c|c|c|c|}
\hline \multirow[t]{5}{*}{ Mediator } & \multicolumn{3}{|c|}{$\begin{array}{l}\text { Mediator concentrations in the aqueous } \\
\text { humor of children with uveitis }\end{array}$} \\
\hline & $\begin{array}{l}\text { Treated with } \\
\text { methotrexate }(n=11)\end{array}$ & $\begin{array}{l}\text { Not treated with } \\
\text { methotrexate }(n=14)\end{array}$ & $p$-Value \\
\hline & Mean $^{a}$ & Mean $^{a}$ & \\
\hline & Range $^{\mathrm{b}}$ & Range $^{\mathrm{b}}$ & \\
\hline & No. ${ }^{c}$ & No. ${ }^{c}$ & \\
\hline \multirow[t]{3}{*}{ IL-1 $\beta$} & 2 & 1 & 0.202 \\
\hline & $1-6$ & $1-7$ & \\
\hline & 5 & 10 & \\
\hline \multirow[t]{3}{*}{ IL-2 } & 60 & 21 & 0.244 \\
\hline & $6-614$ & $6-705$ & \\
\hline & 3 & 6 & \\
\hline \multirow[t]{3}{*}{ IL-4 } & 29 & 9 & 0.344 \\
\hline & $1-1407$ & $1-1407$ & \\
\hline & 4 & 4 & \\
\hline \multirow[t]{3}{*}{ IL-6 } & 74 & 140 & 0.344 \\
\hline & $11-5603$ & $3->13822$ & \\
\hline & 0 & 1 & \\
\hline \multirow[t]{3}{*}{ IL-10 } & 3 & 11 & 0.008 \\
\hline & $1-17$ & $1-114$ & \\
\hline & 4 & 2 & \\
\hline \multirow{2}{*}{$\begin{array}{l}\text { IL-12 } \\
\text { p70 }\end{array}$} & $175-1054$ & $65-988$ & 0.107 \\
\hline & & & \\
\hline \multirow[t]{3}{*}{ IL-13 } & 14 & 7 & 0.317 \\
\hline & $2-104$ & $2-128$ & \\
\hline & 3 & 4 & \\
\hline \multirow[t]{3}{*}{ IL-18 } & 14 & 12 & 0.536 \\
\hline & $3-68$ & $3-72$ & \\
\hline & 2 & 1 & \\
\hline \multirow[t]{3}{*}{ IFN- $\gamma$} & 11 & 4 & 0.183 \\
\hline & $2-104$ & $2-89$ & \\
\hline & 4 & 7 & \\
\hline \multirow[t]{3}{*}{ TNF- $\alpha$} & 9 & 3 & 0.107 \\
\hline & $1-61$ & $1-37$ & \\
\hline & 2 & 4 & \\
\hline \multirow[t]{3}{*}{ sICAM } & 3298 & 4069 & 0.344 \\
\hline & $621->10001$ & $490->10001$ & \\
\hline & 0 & 0 & \\
\hline \multirow[t]{3}{*}{ sVCAM } & 4537 & 4482 & 0.809 \\
\hline & $1286->10105$ & $956->10105$ & \\
\hline & 0 & 0 & \\
\hline \multirow[t]{3}{*}{ RANTES } & 64 & 132 & 0.134 \\
\hline & $9-261$ & $9-1175$ & \\
\hline & 2 & 1 & \\
\hline \multirow[t]{3}{*}{ Eotaxin } & 6 & 6 & 0.809 \\
\hline & $2-18$ & $2-41$ & \\
\hline & 4 & 4 & \\
\hline \multirow[t]{3}{*}{ IL-8 } & 24 & 102 & 0.021 \\
\hline & $5-99$ & $5-1715$ & \\
\hline & 2 & 1 & \\
\hline \multirow[t]{3}{*}{ IP-10 } & 863 & 856 & 0.767 \\
\hline & $204-2018$ & $52-3680$ & \\
\hline & 0 & 0 & \\
\hline
\end{tabular}

IL, interleukin; IFN, interferon; TNF, tumor necrosis factor; sICAM, soluble intercellular adhesion molecule; sVCAM, soluble vascular cell adhesion molecule; RANTES, regulated on activation normal T-cell expressed and secreted; IP, interferon-inducible protein. Sensitivity of the assay is $1.4 \mathrm{pg} / \mathrm{ml}$ for IL- $1 \beta, 1.8$ for IL-2, 1.2 for IL-4, 2.4 for IL-6, 2.3 for IL-10, 4.3 for IL-12 p70, 1.0 for IL-13, 1.2 for IL-18, 9.1 for IFN- $\gamma$, and 1.2 for TNF$\alpha$. Sensitivity for sICAM (CD54) is $26.4 \mathrm{pg} / \mathrm{ml}$, for sVCAM (CD106) 22.3, for RANTES (CCL5) 1.5, for Eotaxin (CCL11) 1.3, for IL-8 (CXCL8) 5.3, and for IP-10 (CXCL10) 1.0 (sensitivity data from de Jager et al., 2005).

a Geometric mean concentration $(\mathrm{pg} / \mathrm{ml})$.

b Range of detectable measured samples (pg/ml).

c Number of samples in the undetectable range. active uveitis were significantly less treated with MTX (0/9) than children with quiet uveitis $(11 / 16, p=0.001)$. The definite diagnosis of patients with diagnostic AqH taps were herpes virus infection $(n=1)$, HLA-B27 $(n=2)$, Devic's neuromyelitis optica $(n=1)$ and unknown etiology $(n=5)$. All active samples, except one, were taken during the first 3 months after the diagnosis of uveitis.

Samples collected during surgery (quiet uveitis samples) had significantly higher levels of IL-2, IL-6, IL-13, IL-18, IFN- $\gamma$, TNF- $\alpha$, sICAM-1, RANTES, IL- 8 and IP-10 than $\mathrm{AqH}$ samples of controls.

Interleukin-10 was also found at a significantly lower level in AqH samples taken after more than 3 months after the diagnosis of uveitis $(n=11)$ than in $\mathrm{AqH}$ samples taken during the first 3 months after the diagnosis of uveitis $(n=10 ; 3 \mathrm{pg} / \mathrm{ml}$ versus $28 \mathrm{pg} / \mathrm{ml}$, respectively, $p=0.016$ ). The first group was significantly more often treated with MTX at time of sample taking $(11 / 15$ versus $0 / 10, p=0.001)$. Of all children in whom the samples were taken during the first 3 months after the diagnosis of uveitis none had JIA-associated uveitis and 11 out of 15 children in the group in whom samples were taken after more than 3 months had JIA-associated uveitis ( $p=$ 0.001 ). All other 15 mediators tested were equal in both groups.

JIA was the most common systemic disease in our population $(n=11)$. Significantly higher levels of IL-2, IL-6, IL-13, IL-18, IFN- $\gamma$, TNF- $\alpha$, sICAM-1, RANTES and IP-10 were found in $\mathrm{AqH}$ of children with JIA-associated uveitis than in controls (Table 2). Interleukin-8 and IL-10 levels in AqH of these children did not differ significantly from controls, whereas these cytokines were significantly increased in the total uveitis group compared with controls. All AqH mediators from children with JIA-associated uveitis were similar to levels measured in children with other uveitis entities $(n=14)$. Children with JIA-associated uveitis were significantly more often treated with MTX $(9 / 11)$ than children with uveitis not associated with JIA $(2 / 14, p=0.001)$. When comparing AqH samples of children with JIA-associated uveitis $(n=11)$ with only those with uveitis of unknown etiology $(n=9)$, no significant differences were found as well.

No differences were found between cytokine levels in $\mathrm{AqH}$ samples of patients with different types of JIA: persistent oligoarticular JIA $(n=6)$, extended oligoarticular JIA $(n=2)$ or polyarticular JIA $(n=3)$.

At the time of sample collection only one girl with JIAassociated uveitis was treated with anti-TNF (etanercept twice a week $12 \mathrm{mg}$ subcutaneously) and MTX. Her TNF level in $\mathrm{AqH}$ was $14 \mathrm{pg} / \mathrm{ml}$.

Repeated samples of the same eye were available for 6 patients (nos. 3, 7, 8, 17, 18 and 25; Table 1). The first samples were taken during surgery $(n=4)$ or for a diagnostic procedure $(n=2)$. All second samples were taken during surgery $(n=6)$. The median time interval between the first and second sample was 1.5 years (range: $0.2-2.3$ years). Significantly higher levels of IL-6 were observed in the first samples compared with the second samples $(122 \mathrm{pg} / \mathrm{ml}$ versus $12 \mathrm{pg} / \mathrm{ml}$; $p=0.046)$. This significant difference was not found when we excluded the diagnostic samples. 


\section{Discussion}

This is the first study that shows that multiple intraocular cytokines, chemokines and soluble adhesion molecules are elevated in $\mathrm{AqH}$ of children with uveitis. The elevated levels were observed for cytokines associated with $\mathrm{T}$ helper 1 (Th1; IL-2, IFN- $\gamma$, TNF- $\alpha$ ), Th2 (IL-13) and T regulatory (Tr; IL-10) response. The reason that we did not find a predominance of Th1 cytokine profile, as previously described in the literature, might be explained by the mixed study population (Lacomba et al, 2000; Takase et al., 2006). However, borderline higher levels of IP-10 and IL-2 were found for the chronic course ( $p=0.068$ and $p=0.069$, respectively). This observation might suggest a predominant Th1 response in children with chronic uveitis. Furthermore, in $\mathrm{AqH}$ of children in whom the samples were collected before start of systemic treatment, we saw higher levels of IL-8, a pro-inflammatory cytokine, compared with $\mathrm{AqH}$ of children in whom treatment with systemic corticosteroids and/or MTX was already started.

Interleukin-8 (CXCL8) is a chemokine with profound effects on neutrophils, is chemotactic for $\mathrm{T}$ lymphocytes and can induce surface expression of adhesion molecules and is known as a pro-inflammatory cytokine (de Vos et al., 1992; Dinarello, 1999; Nussenblatt, 2004). Interleukin-10 is an anti-inflammatory cytokine which prevents for the development of autoimmune diseases (Roncarolo et al., 2001; Thompson and Powrie, 2004). The observation that higher levels of IL-10 and IL-8 were detected in samples collected for diagnostic purpose (active uveitis) compared with samples collected during ocular surgery (quiet uveitis) might implicate that these cytokines are mainly involved in active uveitis. Interleukin-10 was higher in samples taken during the first 3 months after the diagnosis of uveitis. These samples were mainly (8 out of 10) collected for diagnostic purposes before the start of systemic treatment. Therefore IL-10 might be involved in the early stage of the disease process, in active uveitis, or both. The lower levels of IL-8 in uveitis samples collected during ocular surgery compared with diagnostic samples might be explained by the fact that the ocular inflammation was at that point quiet (due to treatment). However, IL8 and IL-10 were found in a lower range in children treated with MTX. From our data it is not clear whether the early stages or active uveitis are responsible for higher levels of IL-8 and IL-10 or, alternatively, whether the absence of treatment with MTX causes this difference.

Despite the absence of clinical signs of active inflammation, elevated cytokine levels were found in the samples obtained during surgical procedures compared with controls. This might implicate that when the uveitis is in clinical remission (no inflammatory cells in the anterior chamber or other inflammatory features), there is an ongoing immune response in the eye based on the elevated cytokines. When comparing mediator levels in $\mathrm{AqH}$ collected during successive surgical interventions in the same eye, no change in (inflammatory) cytokines was found. As such, this is reassuring because it indicates that the surgical intervention itself does not lead to a trauma induced cytokine response.
In the present study we did not find differences in cytokine pattern between the different types of JIA. Patients with systemic JIA have higher serum levels of IL-6 than other forms of JIA (Rooney et al., 1995). Our series does not include samples of uveitis patients with systemic JIA, because uveitis is a very rare feature of systemic JIA. Furthermore, the JIA samples used in this study were collected during cataract or glaucoma surgery. So all these patients had surgery requiring complications of JIA-associated uveitis and therefore they might not be representative of the total JIA-associated uveitis population.

Limitations of this study include the restricted number of samples which precludes meaningful comparison of specific disorders or subcategories of disorders such as anatomic classification, course of uveitis and systemic diseases. Furthermore, our control group was not perfectly age-matched and low in number. The reason for this is that $\mathrm{AqH}$ samples of children with or without uveitis are not readily available. At the time of sample collection the children in the control group were younger than the children with uveitis. However, when we compared our control samples with the adult controls of the study of our colleagues, no major differences were found, except for IL-6, sICAM-1 and sVCAM-1 (van Kooij et al., 2006). More detailed studies on the age dependency of cytokine profiles in $\mathrm{AqH}$ of uveitis patients are currently underway.

For future studies, it will be important to collect and examine larger series of $\mathrm{AqH}$ samples of children with uveitis, in order to make comparisons between different systemic diseases (for example JIA-associated versus HLA-B27 positive uveitis) among the 4 anatomical classifications of uveitis and other comparisons possible. We did not find significant differences between cytokine levels in AqH of JIA-associated uveitis and other uveitis entities. Based on this relatively small study population we cannot conclude whether there is a common pathway in juvenile uveitis, irrespective of the underlying cause. It would be interesting to examine this in a bigger study population with a special regard for IL-8 and IL-10. Furthermore, it would be interesting to compare cytokine and total IgG levels in AqH with those in serum of children with uveitis, to make a discrepancy between local production and influx from elsewhere due to blood aqueous barrier breakdown.

In the last decades the knowledge of the immune system has improved and treatment of inflammatory diseases with biological agents has become available. Biologics are drugs directed against specific cytokines or their receptors (Dunn, 2004). In children, treatment of JIA with anti-TNF has been successful for arthritis. In the near future, treatment with several other biologics might become available. Therefore more insight into the cytokine profile in childhood uveitis is warranted.

In conclusion, our data suggest that a spectrum of cytokines, chemokines and soluble adhesion molecules in the $\mathrm{AqH}$ of children with uveitis are involved in the pathogenesis of uveitis. These mediators are present irrespective of active or inactive uveitis and without clear predominance of a Th1 or Th2 associated cytokine profile. Whether the IL-10 and IL-8 
levels in AqH appear to be related to uveitis activity, early or late phase of the course of uveitis or systemic treatment with MTX needs further investigation. Further studies will be required to elucidate the exact role of these mediators in the pathogenesis of different uveitis entities, especially JIA, and specific treatment strategies.

\section{Acknowledgement}

K.M.S. was supported by the Dr. F.P. Fischer Foundation, Utrecht, The Netherlands.

\section{References}

Curnow, S.J., Falciani, F., Durrani, O.M., 2005. Multiplex bead immunoassay analysis of aqueous humor reveals distinct cytokine profiles in uveitis. Invest. Ophthalmol. Vis. Sci. 46, 4251-4259.

de Boer, J., Wulffraat, N., Rothova, A., 2003. Visual loss in uveitis of childhood. Br. J. Ophthalmol. 87, 879-884

de Groot-Mijnes, J.D.F., Rothova, A., van Loon, A.M., et al., 2006. Polymerase chain reaction and Goldmann-Witmer coefficient analysis are complementary for the diagnosis of infectious uveitis. Am. J. Ophthalmol. 141, 313-318.

de Jager, W., Prakken, B.J., Bijlsma, J.W., et al., 2005. Improved multiplex immunoassay performance in human plasma and synovial fluid following removal of interfering heterophilic antibodies. J. Immunol. Methods 300, 124-135.

de Vos, A.F., Hoekzema, R., Kijlstra, A., 1992. Cytokines and uveitis, a review. Curr. Eye Res. 11, 581-597.
Dinarello, C.A., 1999. IL-18: A TH1-inducing, proinflammatory cytokine and new member of the IL-1 family. J. Allergy Clin. Immunol. 103, $11-24$.

Dunn, J.P., 2004. Review of immunosuppressive drug therapy in uveitis. Curr. Opin. Ophthalmol. 15, 293-298.

Evereklioglu, C., 2005. Current concepts in the etiology and treatment of Behcet disease. Surv. Ophthalmol. 50, 297-350.

Kanski, J.J., Shun-Shin, G.A., 1984. Systemic uveitis syndromes in childhood: an analysis of 340 cases. Ophthalmology 91, 1247-1252.

Lacomba, M.S., Martin, C.M., Chamond, R.R., et al., 2000. Aqueous and serum interferon gamma, interleukin (IL) 2, IL-4, and IL-10 in patients with uveitis. Arch. Ophthalmol. 118, 768-772.

Nussenblatt, R.B., 2004. Uveitis. Fundamentals and Clinical Practice, third ed. Elsevier, Philadelphia, PA.

Roncarolo, M.G., Bacchetta, R., Bordignon, C., et al., 2001. Type 1 T regulatory cells. Immunol. Rev. 182, 68-79.

Rooney, M., David, J., Symons, J., et al., 1995. Inflammatory cytokine responses in juvenile chronic arthritis. Br. J. Rheumatol. 34, 454-460.

Rothova, A., Suttorp-van Schulten, M.S., Treffers, W.F., Kijlstra, A., 1996. Causes and frequency of blindness in patients with intraocular inflammatory disease. Br. J. Ophthalmol. 80, 332-336.

Takase, H., Futagami, Y., Yoshida, T., et al., 2006. Cytokine profile in aqueous humor and sera of patients with infectious or noninfectious uveitis. Invest. Ophthalmol. Vis. Sci. 47, 1557-1561.

Thompson, C., Powrie, F., 2004. Regulatory T cells. Curr. Opin. Pharmacol. 4, $408-414$.

Tugal-Tutkun, I., Havrlikova, K., Power, W.J., Foster, C.S., 1996. Changing patterns in uveitis of childhood. Ophthalmology 103, 375-383.

van der Lelij, A., Rothova, A., 1997. Diagnostic anterior chamber paracentesis in uveitis: a safe procedure? Br. J. Ophthalmol. 81, 976-979.

van Kooij, B., Rothova, A., Rijkers, G.T., de Groot-Mijnes, J.D., 2006. Distinct cytokine and chemokine profiles in the aqueous of patients with uveitis and cystoid macular edema. Am. J. Ophthalmol. 142, 192-194. 